\title{
Three Dimensional Analysis of Phase Transformations in Ferrous Alloys
}

\author{
G. Spanos*, and D.J. Rowenhorst** \\ * Physical Metallurgy Branch, Code 6324, Naval Research Laboratory, Washington, DC, 20375 \\ ** National Research Council, Washington, DC, 20001 (stationed at the Naval Research \\ Laboratory)
}

Despite the importance of understanding the true three dimensional (3D) morphology, distribution, crystallography, and interconnectivity of solid-state precipitates and matrix grains, microstructural characterization prior to the last decade has usually been accomplished by methods that enable only two dimensional (2D) observations. This talk will provide a brief overview of how these 2D techniques have been expanded to encompass 3D microstructural investigations at the Naval Research Laboratory (NRL) that have resulted in many new findings in four different ferrous-based alloy systems.

Computer-aided three dimensional (3D) reconstruction of serial sections of an Fe-C-Ni alloy were used to determine the three-dimensional shapes and distributions of grain boundary nucleated proeutectoid ferrite precipitates (Figure 1), which appeared as both Widmanstätten shapes and more equiaxed grain boundary precipitates. While the two dimensional (2D) cross-sections of these precipitates "appeared" to fit into the previously accepted categories of precipitate morphologies, 3D reconstructions revealed important aspects of connectivity and shape that were not observed earlier [1].

A major part of this presentation will concentrate on integrating 3D reconstructions from optical micrographs with Electron Back-Scattered Diffraction (EBSD) to determine the 3D morophology and crystallographic relationships in coarse autotempered martensite formed in a high strength low alloy steel (HSLA-100). This microstructure can be formed readily through a simple continuous cooling cycle, and it auto-tempers to form a dispersion of fine carbides, generating the potential for attractive properties. Emphasis will be placed on some new techniques being employed at the Naval Research Laboratory (NRL) to combine in a quantitative fashion morphological and crystallographic observations taken from multiple serial sections, and then applied to $3 \mathrm{D}$ reconstructions of this microconstituent. These results include "3D Interface Orientation Histograms" which quantify the crystallographic direction of interface normals (and/or growth directions) in these crystals, particularly highlighting various 3D facets. The 3D Interface Orientation Histograms are somewhat analogous to two dimensional (2D) inverse pole figures taken from individual 2D planes of polish by conventional Electron Backscatter Diffraction (EBSD) analyses. These observations are further quantified by reporting the average 3D habit plane of various facets on these crystals.

Finally, a demonstration of how these techniques are being applied to austenite and sigma phase microstructures in a superaustenitic stainless steel will be provided (e.g., see Fig. 2).

[1.] M. V. Kral and G. Spanos, in press, Metallurgical and Materials Transactions (2005). 

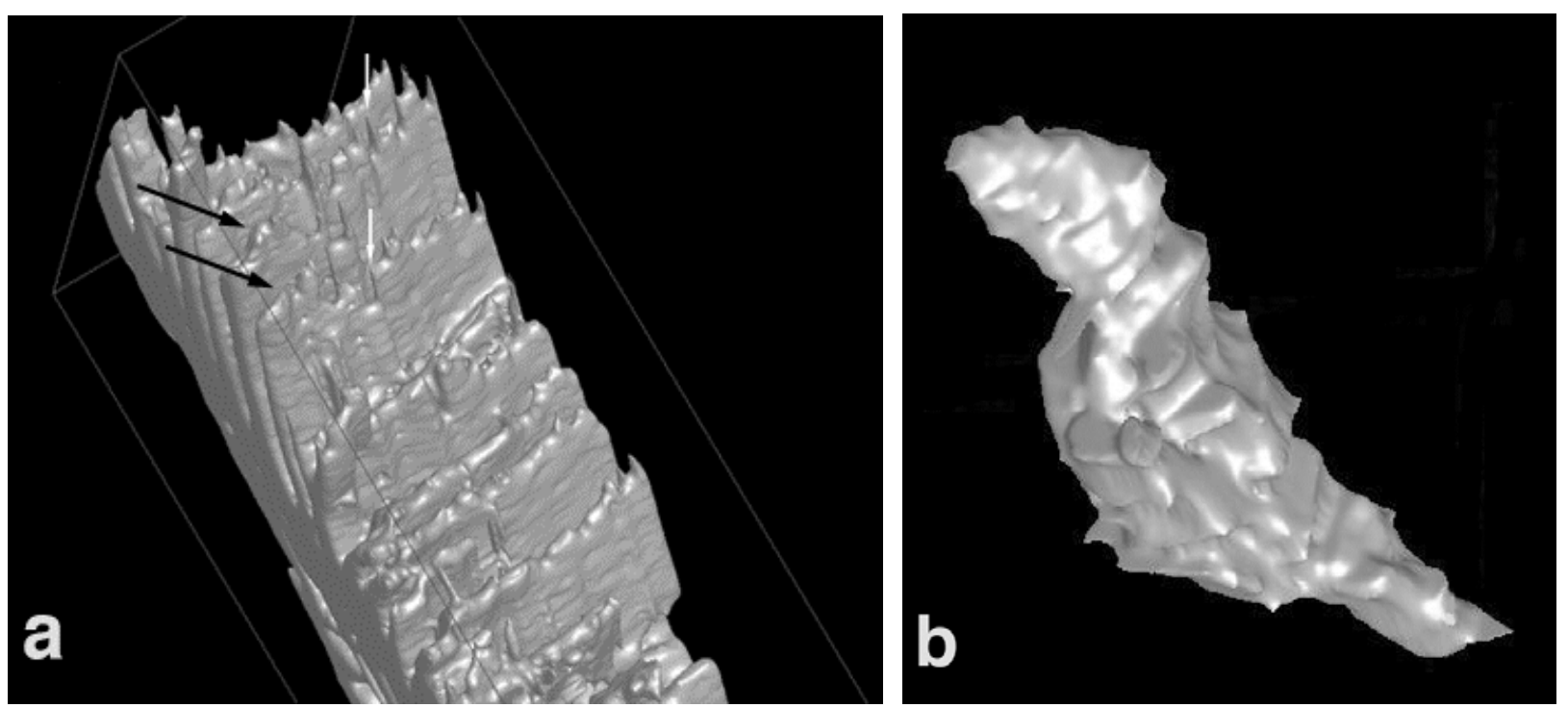

Figure 1. Typical computer reconstructions of (a) plate-like ferrite precipitates, and (b) a grain boundary allotriomorph in a Fe-C-Ni alloy generated from serial sections. Taken from [1].

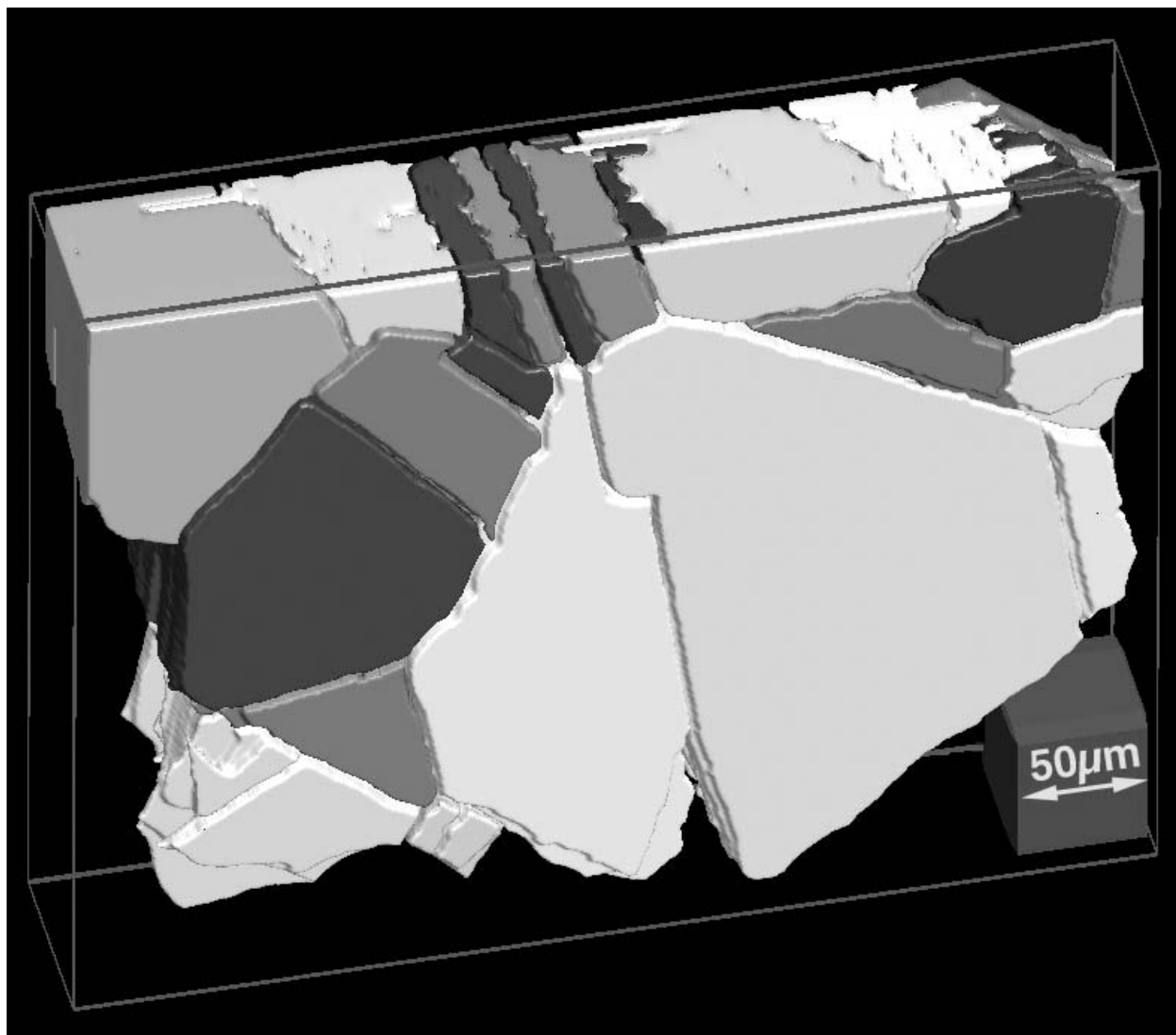

Figure 2. 3D reconstructions of austenite grains in an austenitic stainless steel (courtesy of A. Lewis and A. Geltmacher, Naval Research Laboratory). 